\title{
Assessment of premenstrual syndrome among reproductive aged Myanmar women
}

\author{
Htet Htet Oo', Mya Thanda Sein², Ohn Mar', Aye Aung ${ }^{4}$ \\ ${ }^{1}$ Lecturer, Department of Physiology, University of Medicine 2, Yangon, Myanmar, ${ }^{2}$ Professor and Head, Department of \\ Physiology, University of Medicine Magway, Myanmar, ${ }^{3}$ Professor and Head, Department of Physiology, University of \\ Medicine 1, Yangon, Myanmar, ${ }^{4}$ Rector, University of Medicine 2, Yangon, Myanmar
}

Background: Almost all women experience at least mild degree of one or more premenstrual symptoms during the days before menstruation. Women with premenstrual syndrome (PMS) experience a pattern of severe premenstrual symptoms month after month and premenstrual dysphoric disorder (PMDD) is the extreme, predominantly psychological end of the PMS spectrum. These symptoms hinder some aspect of their family as well as social lives and also interfere their activities at work. Aims and Objectives: This study aimed to assess the prevalence of PMS among reproductive aged Myanmar women from medical field and to investigate the frequency and severity of symptoms experience by the women with PMS. Materials and Methods: All participants were asked to complete a questionnaire derived from Calendar of Premenstrual Experiences (COPE) for two consecutive menstrual cycle to diagnose PMS and PMDD. A cross-sectional descriptive study was conducted in 400 reproductive aged women (between 15 - 45 years) among the medical students, doctors and nurses. Results: Out of 400 subjects, 149 women (37.3\%) met DSM-IV criteria for PMS and 251 women (62.7\%) did not have PMS. Among PMS women, 81 women (54.4\%) had PMDD. The most frequent PMS symptoms were poor concentration (88.6\%), irritability $(87.3 \%)$, ache and pain $(81.9 \%)$, increased appetite $(79.2 \%)$ and breast swelling $(75.8 \%)$. The most frequent symptom in mild and moderate PMS is physical symptom (ache and pain) and that in PMDD was psychological symptom (poor concentration). Conclusion: The prevalence of PMS with high PMDD proportion was found in reproductive aged Myanmar women from medical field.

Key words: Premenstrual symptoms, Premenstrual syndrome (PMS), Premenstrual dysphoric disorder (PMDD), Calendar of premenstrual experiences (COPE)

\section{Access this article online}

Website:

http://nepjol.info/index.php/AJMS DOI: 10.3126/ajms.v7i4.13298 E-ISSN: 2091-0576 P-ISSN: 2467-9100

\section{INTRODUCTION}

During reproductive age, women generally experience physical, psychological and behavioral symptoms regularly at the same phase of each menstrual cycle, especially during the premenstrual period or early menstruation, but occasionally symptoms occur at ovulation. Premenstrual syndrome (PMS) is a condition which manifests with distressing physical, behavioral and psychological symptoms, in the absence of organic or underlying psychiatric disease, which regularly recurs during the luteal phase of each menstrual cycle and which disappears or significantly regresses by the end of menstruation. ${ }^{1}$ The onset of the menstrual flow usually brings dramatic and complete relief of these symptoms. According to American College of Obstetricians and Gynecologists (ACOG) (2009), at least $(85 \%)$ of the menstruating women have experienced at least one premenstrual symptom and these symptoms may appear to some degree at the different stages in their reproductive lives. ${ }^{2}$

The degree and type of the premenstrual symptoms can vary significantly from woman to woman but their character is less important than their timing and their severity. In PMS, the premenstrual symptoms can affect work and school performance, and lead to problems/ 
conflicts in interpersonal relation-ships. It was noted that the cyclical luteal timing and the severity are important to evaluate for the diagnosis of PMS. ${ }^{3}$ Premenstrual dysphoric disorder (PMDD) is the extreme, predominantly psychological end of the PMS spectrum. ${ }^{1}$ In cases of PMDD, symptoms become severe enough to cause severe disability and often require more aggressive treatment that requires pharmacological intervention in addition to nonpharmacological treatments.

Mortola et al. (1990) designed the Calendar of Premenstrual Experience (COPE), a calendar form single sheet diary, and also assessed its validity and reliability for diagnosis of PMS. ${ }^{4}$ It is a list of 21 premenstrual symptoms to measure psychological, physical and behavioral factors prospectively for each day of the menstrual cycle. The premenstrual symptoms are recorded by self-report visual analogue scales. The scoring in COPE is 3- point rating scale for each symptom: absent (0), present (1) and severe enough to disturb daily life in various aspects (2). They showed that the COPE distinguished women with PMS from controls correctly with $2.8 \%$ false-negative rate and no false positives when used for two consecutive menstrual cycles. Similarly Michael and Shaw (2002) assessed premenstrual symptoms with COPE in 215 PMS patients over two consecutive months and also recommended that the COPE is valid and reliable instrument for identifying PMS. ${ }^{5}$ The COPE is the most acceptable practical record applicable in clinical and research settings.

PMS has been studied and evaluated extensively in the Western countries, but only a limited amount of research studies have been conducted in Asia. In Myanmar, women suffering premenstrual symptoms regard them as common or trivial problems not requiring medical attention. In order to increase awareness of the unique pathophysiological nature of menstruation related symptoms and to promote the health and well-being of reproductive age women, this study aimed to assess the prevalence of PMS among the reproductive aged Myanmar women and investigate the frequency and severity of symptoms experience by the women with PMS.

\section{MATERIALS AND METHODS}

\section{Selection of participants}

This cross-sectional descriptive study was conducted in the Department of Physiology, University of Medicine 2, Yangon, Myanmar. A total of 400 apparently healthy reproductive aged (between 15 to 45years) female medical students, doctors and nurses from the University of Medicine 1 and 2, Yangon and from Muslim Free Hospital, Yangon were recruited. A written informed consent was obtained from all volunteered subjects individually.
The Board of postgraduate studies of the University of Medicine 2, Yangon approved the study protocol. Prescreening evaluation including medical, menstrual, gynecological and obstetrics history taking and thorough physical examination was done according to Pro forma. The subjects who have pregnancy, contraception, amenorrhea and current medical, psychiatric or gynecological problems were excluded from the study.

\section{Study design}

The subjects were explained how to fill up the selfreported COPE form and how to take and record basal body temperature throughout the menstrual cycle. They were instructed to take oral temperature measurement immediately after waking up, before taking any hot/cold drink in the morning. In the evening, each subject have to record her experience of each and every symptom in COPE during the previous 12-18 hours as mild, moderate, severe or totally absent. For monitoring, they were instructed to inform and meet the investigator after completing questionnaire for first menstrual cycle. On the check-up visit, the investigator collected the questionnaires they filled up, reviewed them and explained the errors if necessary. Then, they filled up the form for next consecutive menstrual cycle. At the end of the second menstrual cycle, all the questionnaires were collected and the assessment of PMS was done.

\section{Assessment of PIMS}

According to the ACOG (2000), criteria for diagnosis of PMS includes at least one moderate to severe mood symptom and one physical symptom, and criteria for the diagnosis of PMDD includes a total of five symptoms with one severe mood symptom. ${ }^{5}$ Mood or affective symptoms are irritability, depression, tearfulness, anxiety, tension, confusion, uncontrollable anger and loneliness. While in somatic pain symptom, there are headache, migraine, breast tenderness, back pain, abdominal cramps, general pain. Others are fluid retention symptoms such as weight gain, abdominal bloating, edema of extremities, abdominal swelling and food symptoms including increased appetite, specific food cravings and nausea. It has been reported up to 200 premenstrual symptoms of varying degrees of severity. ${ }^{4}$

The assessment of PMS was done by prospective symptom screening with COPE for two consecutive menstrual cycles according to DSM-IV criteria. Women were considered having PMS if they present with at least one severe (severity rating score $=2$ ) psychological symptom (Item 1 to 8 of the COPE) and one severe (severity rating score $=2$ ) physical symptom (Item 9 to 13 of the COPE) in the luteal phase of the menstrual cycle with free of these symptoms in the follicular phase. Those present with a 
total of five symptoms including one severe (severity rating score $=2$ ) mood symptom (Item 1 to 4 of the COPE) in the luteal phase of the menstrual cycle with free of these symptoms in the follicular phase were considered having severe PMS or premenstrual dysphoric disorder (PMDD). Those present with PMS defining symptoms which are not severe enough to include PMDD criteria are considered as mild and moderate PMS. Women with no symptom or mild (severity rating score $=1$ ) symptoms included in without PMS group.

\section{Statistical analysis}

The presence or absence of PMS, the frequencies and severity of the PMS symptoms experienced by the subjects were calculated in frequency and percentage.

\section{RESULTS}

Out of 400 subjects assessed for PMS, 149 women (37.3\%) met DSM-IV criteria for PMS and 251 women (62.7\%) did not have PMS. The (54.4\%) of women with PMS (81 women) have premenstrual symptoms severe enough to induce impairment and distress, so they met strict criteria for PMDD. Therefore, the $20.3 \%$ of total subjects had PMDD (Figure 1).

The presenting premenstrual symptoms of the subjects were listed in the Table 1. Five most prevalent symptoms of all women with PMS were poor concentration (88.4\%), irritability $(87.1 \%)$, ache and pain $(81.7 \%)$, increased appetite $(79.0 \%)$ and breast swelling $(75.7 \%)$. Only fourteen (9.4\%) subjects complained uncontrollable anger which was the least prevalent symptom in women with PMS.

Five most prevalent symptoms of women with mild and moderate PMS were ache and pain (85.3\%), irritability $(79.4 \%)$, poor concentration $(79.4 \%)$, food craving $(76.5 \%)$ and abdominal bloating $(72.1 \%)$. None of the women with mild and moderate PMS complained uncontrollable anger.

Five most prevalent symptoms of women with PMDD were poor concentration $(96.3 \%)$, anxiety $(95.1 \%)$, breast

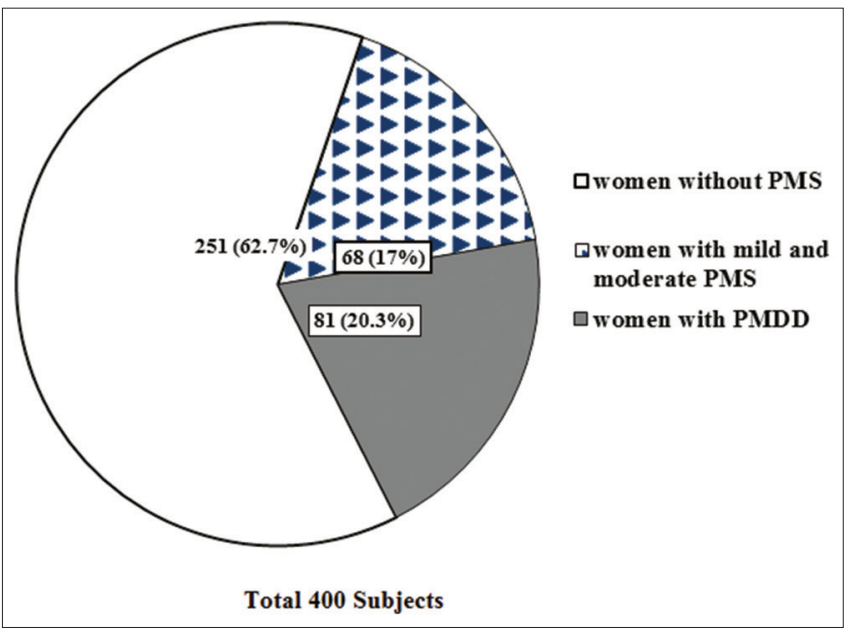

Figure 1: Prevalence of PMS and PMDD in studied population

\section{Table 1: Presenting symptoms of women with premenstrual syndrome}

\begin{tabular}{|c|c|c|c|c|}
\hline No & Complained symptoms & PMS (n=149) & PMDD (n=81) & Mild and moderate PMS ( $n=68)$ \\
\hline I & Psychological symptoms & & & \\
\hline 1 & Depression & 43.0 & 49.4 & 36.8 \\
\hline 2 & Anxiety & 70.4 & 95.1 & 41.2 \\
\hline 3 & Mood swing & 44.9 & 61.7 & 25.0 \\
\hline 4 & Irritability & 87.1 & 93.8 & 79.4 \\
\hline 5 & Anger & 59.6 & 76.5 & 39.7 \\
\hline 6 & Poor concentration & 88.4 & 96.3 & 79.4 \\
\hline 8 & Lethargy & 64.3 & 77.8 & 48.5 \\
\hline II & Physical symptoms & & & \\
\hline 9 & Breast swelling/tenderness & 75.7 & 95.1 & 52.9 \\
\hline 10 & Headache & 56.2 & 65.4 & 45.6 \\
\hline 11 & Ache and pain & 81.7 & 79.0 & 85.3 \\
\hline 12 & Abdominal bloating & 75.0 & 77.8 & 72.1 \\
\hline 13 & Swelling in hands and feet & 31.5 & 42.0 & 19.1 \\
\hline 15 & Food craving & 60.0 & 48.2 & 76.5 \\
\hline 16 & Hypersomnia & 62.0 & 76.5 & 45.6 \\
\hline 17 & Insomnia & 34.2 & 48.2 & 17.7 \\
\hline 18 & Short temper & 64.3 & 64.2 & 64.7 \\
\hline 19 & Uncontrollable anger & 9.4 & 17.3 & 00.0 \\
\hline 20 & Apathy & 45.6 & 30.9 & 63.2 \\
\hline 21 & Avoidance of social contact & 55.6 & 64.2 & 45.6 \\
\hline \multicolumn{5}{|c|}{ Percent $(\%)$ of women } \\
\hline
\end{tabular}




\begin{tabular}{|c|c|c|c|c|c|}
\hline & No & Age & Study population & PMS (\%) & PMDD (\%) \\
\hline Takeda et al., (2006) & 1187 & $20-49$ & Japanese women & 5.3 & 1.2 \\
\hline Umoiyoho et al., (2006) & 200 & $16-31$ & Nulliparous students of the University of Calabar, Nigeria & 85.5 & - \\
\hline Chayachinda et al., 2008 & 423 & - & Thai nurses & 25.1 & - \\
\hline HlaHla Aye et al., (1995) & 102 & $21 \pm 1$ & $2^{\text {nd }}$ year MBBS students in UM 1, Yangon & 74.5 & - \\
\hline Khin Than Aye (2001) & 300 & $15-29$ & Medical students in Institute of Medicine, Mandalay & 80.6 & - \\
\hline Present study & 400 & $15-45$ & $\begin{array}{l}\text { Medical students, doctors and nurses in UM } 1 \text { \& 2, } \\
\text { Yangon and in MFH }\end{array}$ & 37.3 & 20.3 \\
\hline
\end{tabular}

swelling (95.1\%), increased appetite (95.1\%) and irritability $(93.8 \%)$. Seventy eight $(96.3 \%)$ subjects complained poor concentration which was the most prevalent symptom. Fourteen (17.3\%) women with PMDD complained uncontrollable anger which was the least prevalent symptom.

\section{DISCUSSION}

According to ACOG (2009), at least (85\%) of the women who menstruate have experienced at least one premenstrual symptom and the $(30 \%)$ to $(40 \%)$ of menstruating women have severe enough symptoms to disrupt their daily lives (PMS). ${ }^{2}$ The prevalence of PMS in the present study (i.e. $37.3 \%$ ) was in agreement with the ACOG report.

The present study also noted that $54.4 \%$ of the PMS women had PMDD. ACOG reported that (3\%) to $(8 \%)$ of PMS women have severely distressing symptoms (PMDD). ${ }^{2}$ The prevalence of PMDD among PMS women observed in the present study was greater than the PMDD report of ACOG. However, the prevalence of PMDD among reproductive aged women observed in the present study (i.e. $20.3 \%$ ) was in accordance with the RCOG report. RCOG guideline (2007) has reported that the prevalence of PMDD is variable between $(3 \%)-(30 \%)$ of the menstruating women. ${ }^{1}$

The prevalence of PMS in this study was found to be high, considering the previous reports of two Asian studies. In the assessment of PMS in 1187 Japanese women between the ages of 20 to 49 years by using the premenstrual menstrual symptom questionnaire, the prevalence of PMS and PMDD were $(5.3 \%)$ and $(1.2 \%)$ respectively. ${ }^{6}$ Another Asian study conducted in 423 Thai nurses from a University Hospital during October 2005 to March 2006 documented the prevalence of PMS as (25.1\%). ${ }^{7}$ But the prevalence of PMS in the present study (37.3\%) was much lower than that of the previous study which reported that $(85.5 \%)$ of 200 nulliparous students between the ages of 16 to 31 years from the University of Calabar, Nigeria regularly experienced symptoms suggestive of PMS. ${ }^{8}$
Previously, two Myanmar research have studied the PMS in medical students. The (74.5\%) of 102 second year MBBS students attending in the Institute of Medicine 1, Yangon, had PMS (Hla-Hla-Aye, Myint-Aye-Mu, ChoCho-Yu-Mon and Myo-Khin, 1995). .' Another study also reported that the prevalence of PMS in medical students attending in the Institute of Medicine, Mandalay during the study period of $1^{\text {st }}$ February 2000 to $31^{\text {st }}$ July 2000 was $(80.6 \%) .{ }^{10}$ Considering the findings of all these studies, the prevalence of PMS varies a lot. One possibility for the great variability in the PMS prevalence among these studies was PMS questionnaires utilized in these studies. The present study used COPE to find out PMS but the others used different questionnaires. Difference in study population was also considered as the explanation for the discrepancy in the prevalence.

In a study done by Navdeep (2009), 197 students (79.43\%) had pain in lower abdomen, 164 students $(66.12 \%)$ had backache, 160 students (64.51\%) had irritability, 113 students $(45.05 \%)$ had pain in thighs and 73 students $(29.43 \%)$ had pain in breast. ${ }^{11}$ In this study, the most prevalent symptom of women with PMS ( $\mathrm{n}=149)$ as well as women with PMDD ( $\mathrm{n}=81)$ was poor concentration. The least prevalent symptom was uncontrollable anger. Both were psychological symptoms. However, in women with mild and moderate PMS ( $n=68)$, the most prevalent symptom was physical symptoms (ache and pain). These results showed that the psychological symptom (poor concentration) was most prevalent in women with PMDD and the physical symptom (ache and pain) was most prevalent in women with mild and moderate PMS. Being the extreme, predominantly psychological end of the PMS spectrum, PMDD was presented with more psychological symptoms. The least prevalent symptom, uncontrollable anger was the symptom found only in women with PMDD and none of the women with mild and moderate PMS complained it.

\section{CONCLUSION}

The present study has found that the prevalence of PMS in medical students, doctors and nurses was (37.3\%) with high 
prevalence of PMDD (i.e. 54.4\%) among PMS women. The study population being personnel from medical profession, the present findings suggested that stressful lifestyle might be a factor attributable to PMDD. Future research should to be carried out whether measures to reduce stress would have the beneficial effect on the occurrence of PMDD or not.

\section{REFERENCES}

1. Royal College of Obstetricians and Gynecologists. Management of Premenstrual Syndrome, London: Guideline and Audit Committee of the RCOG, Green-top Guideline No.48: December 2007.

2. American College of Obstetricians and Gynecologists (2009) Patient Education Pamphlet, Women's Health Care Physicians: Premenstrual Syndrome [online] Washington, DC: ACOG. Available from: < http://www. acog.org/publications/patienteducation/bp057.cfm> [Accessed 13 February 2009]

3. Abraham GE. Nutritional factors in the etiology of premenstrual tension syndromes. JRM 1983; 28: 446-464.

4. Mortola JF, Girton L, Beck L and Yen SS. Diagnosis of premenstrual syndrome by a simple, prospective, and reliable instrument: the calendar of premenstrual experiences. Obstetrics and Gynecology 1990; 76(2): 302-307.

5. Michael $F$ and Shaw WS. Measurement properties of the calendar of premenstrual experience in patients with premenstrual syndrome. JRM 2002; 47(4): 279-289.

6. Takeda T, Tasaka K, Sakata M and Murata Y. Prevalence of premenstrual syndrome and premenstrual dysphoric disorder in Japanese women. Archives of Women's Mental Health 2006; 9(4): 209-212.

7. Chayachinda $\mathrm{C}$, Rattanachaiyanont $\mathrm{M}$, Phattharayuttawat $\mathrm{S}$ and Kooptiwoot S. Premenstrual Syndrome in Thai Nurses. JPOG 2008; 29(3): 203-209.

8. Umoiyoho AU, Antai AB, Udezi AW, Ekanem EE and Okon UJ. Premenstrual syndrome: prevalence in students of the University of Clalbar, Nigeria. AJBR 2004; 7: 45-50.

9. Hla-Hla-Aye, Myint-Aye-Mu, Cho-Cho-Yu-Mon and MyoKhin. Hormonal profile comparison of women with menstrual symptoms and those without, in a population of medical students. MMJ, Conference Issue, 1995; 34-37.

10. Khin-Than-Aye. Premenstrual syndrome among medical students of Institute of Medicine Mandalay. [dissertation]. University of Medicine Mandalay, 2001.

11. Navdeep K and Ramesh T. A descriptive study to assess the premenstrual syndrome and coping behaviour among nursing students, NMRJ, National Institute of Nursing Education, PGIMER, Chandigarh 2009; 5 (1): 19-23.

Authors Contribution:

HHO - Conceptualized and executed the study, literature search, collected data and statistically analyzed and interpreted, prepared first draft of manuscript;

MTS - Concept and design of the study, reviewed the literature, statistical analysis and Interpretation of data, participated in drafting, reviewing, and revising the manuscript for intellectual content, approved the manuscript to be published; Oh - Concept and design of the study, review of study, critical revision of the manuscript, approved the manuscript to be published; AA - Concept and design of the study, critical revision of the manuscript, approved the manuscript to

be published.

Source of Support: Nil. Conflict of Interest: None. 